\title{
KOSZUL HOMOLOGY AND SYZYGIES OF VERONESE SUBALGEBRAS
}

\author{
WINFRIED BRUNS, ALDO CONCA, AND TIM RÖMER
}

To Jürgen Herzog, friend and teacher

\begin{abstract}
A graded $K$-algebra $R$ has property $N_{p}$ if it is generated in degree 1 , has relations in degree 2 and the syzygies of order $\leq p$ on the relations are linear. The GreenLazarsfeld index of $R$ is the largest $p$ such that it satisfies the property $N_{p}$. Our main results assert that (under a mild assumption on the base field) the $c$-th Veronese subring of a polynomial ring has Green-Lazarsfeld index $\geq c+1$. The same conclusion also holds for an arbitrary standard graded algebra, provided $c \gg 0$.
\end{abstract}

\section{INTRODUCTION}

A classical problem in algebraic geometry and commutative algebra is the study of the equations defining projective varieties and of their syzygies. Green and Lazarsfeld [18, 19] introduced the property $N_{p}$ for a graded ring as an indication of the presence of simple syzygies. Let us recall the definition. A finitely generated $\mathbb{N}$-graded $K$-algebra $R=\oplus_{i} R_{i}$ over a field $K$ satisfies property $N_{0}$ if $R$ is generated (as a $K$-algebra) in degree 1 . Then $R$ can be presented as a quotient of a standard graded polynomial ring $S$ and one says that $R$ satisfies property $N_{p}$ for some $p>0$ if $\beta_{i, j}^{S}(R)=0$ for $j>i+1$ and $1 \leq i \leq p$. Here $\beta_{i, j}^{S}(R)$ denote the graded Betti numbers of $R$ over $S$. For example, property $N_{1}$ means that $R$ is defined by quadrics, $N_{3}$ means that $R$ is defined by quadrics and that the first and second syzygies of the quadrics are linear. We define the Green-Lazarsfeld index of $R$, denoted by index $(R)$, to be the largest $p$ such that $R$ has $N_{p}$, with index $(R)=\infty$ if $R$ satisfies $N_{p}$ for every $p$. It is, in general, very difficult to determine the precise value of the Green-Lazarsfeld index. Important conjectures, such as Green's conjecture on the syzygies of canonical curves [14, Chap.9], predict the value of the Green-Lazarsfeld index for certain families of varieties.

The goal of this paper is to study the Green-Lazarsfeld index of the Veronese embeddings $v_{c}: \mathbb{P}^{n-1} \rightarrow \mathbb{P}^{N}$ of degree $c$ of projective spaces and, more generally, of the Veronese embeddings of arbitrary varieties. Let $S$ denote the polynomial ring in $n$ variables over the field $K$. The coordinate ring of the image of $v_{c}$ is the Veronese subring $S^{(c)}=\bigoplus_{i \in \mathbb{N}} S_{i c}$ of $S$. If $n \leq 2$ or $c \leq 2$ then $S^{(c)}$ is a determinantal ring whose resolution is well understood and the Green-Lazarsfeld index can be easily deduced. If $n=2$ then $S^{(c)}$ is resolved by the Eagon-Northcott complex and so index $\left(S^{(c)}\right)=\infty$. The resolution of $S^{(2)}$ in characteristic 0 is described by Jozefiak, Pragacz and Weyman in [22]; it follows that index $\left(S^{(2)}\right)=5$ if $n>3$ and $\operatorname{index}\left(S^{(2)}\right)=\infty$ if $n \leq 3$. For $n \leq 6$ Andersen [1] proved that index $\left(S^{(2)}\right)$ is independent on $\operatorname{char} K$, but for $n>6$ and $\operatorname{char} K=5$ she proved that index $\left(S^{(2)}\right)=4$. 
For $n>2$ and $c>2$ it is known that

$$
c \leq \operatorname{index}\left(S^{(c)}\right) \leq 3 c-3 .
$$

The lower bound is due to Green [17] (and holds for any $c$ and $n$ ). Ottaviani and Paoletti [23] established the upper bound in characteristic 0 . They also showed that index $\left(S^{(c)}\right)=$ $3 c-3$ for $n=3$ and conjectured that index $\left(S^{(c)}\right)=3 c-3$ holds true for arbitrary $n \geq 3$; see also Weyman [29, Proposition 7.2.8]. For $n=4$ and $c=3$ it is indeed the case [23, Lemma 3.3]. In their interesting paper [13] Eisenbud, Green, Hulek and Popescu reproved some of these statements using different methods. Rubei [28] proved that index $\left(S^{(3)}\right) \geq 4$ if $\operatorname{char} K=0$. Our main results are the following:

(i) $c+1 \leq \operatorname{index}\left(S^{(c)}\right)$ if char $K=0$ or $>c+1$; see Corollary 4.2.

(ii) If $R$ is a quotient of $S$ then $\operatorname{index}\left(R^{(c)}\right) \geq \operatorname{index}\left(S^{(c)}\right)$ for every $c \geq \operatorname{slope}_{S}(R)$; see Theorem 5.2, In particular, if $R$ is $\operatorname{Koszul}$ then index $\left(R^{(c)}\right) \geq \operatorname{index}\left(S^{(c)}\right)$ for every $c \geq 2$,

Furthermore we give characteristic free proofs of the bounds (11) and of the equality for $n=3$; see Theorem 4.7. Our approach is based on the study of the Koszul complex associated to the $c$-th power of the maximal ideal. Let $R$ be a standard graded $K$-algebra with maximal homogeneous ideal $\mathfrak{m}$. Let $K\left(\mathfrak{m}^{c}, R\right)$ denote the Koszul complex associated to $\mathfrak{m}^{c}, Z_{t}\left(\mathfrak{m}^{c}, R\right)$ the module of cycles of homological degree $t$ and $H_{t}\left(\mathfrak{m}^{c}, R\right)$ the corresponding homology module. In Section 2 we study the homological invariants of $Z_{t}\left(\mathfrak{m}^{c}, R\right)$. Among other facts, we prove, in a surprisingly simple way, a generalization of Green's theorem [17, Thm. 2.2] to arbitrary standard graded algebras; see Corollary 2.5. If $R$ is a polynomial ring (or just a Koszul ring), then it follows that the regularity of $Z_{t}\left(\mathfrak{m}^{c}, R\right)$ is $\leq t(c+1)$; see Proposition 2.4.

In Section 3 we investigate more closely the modules $Z_{t}\left(\mathfrak{m}^{c}, S\right)$ in the case of a polynomial ring $S$. Lemma 3.4 describes certain cycles which then are used to prove a vanishing statement in Theorem 3.6. In Section 4 we improve the lower bound (11) by one, see Corollary 4.2. Proposition 4.4 states a duality of Avramov-Golod type, which is the algebraic counterpart of Serre duality. The duality is then used to establish Ottaviani and Paoletti's upper bound index $\left(S^{(c)}\right) \leq 3 c-3$ in arbitrary characteristic (Theorem 4.7). We also show that for $n=3$ one gets index $\left(S^{(c)}\right)=3 c-3$ independently of the characteristic; see Theorem 4.7 .

In Section 5 we take $R$ to be a quotient of a Koszul algebra $D$ and prove that for every $c \geq \operatorname{slope}_{D}(R)$ we have index $\left(R^{(c)}\right) \geq \operatorname{index}\left(D^{(c)}\right)$; see Theorem 5.2. Here slope $(R)=$ $\sup \left\{t_{i}^{D}(R) / i: i \geq 1\right\}$ where $t_{i}^{D}(R)$ is the largest degree of an $i$-th syzygy of $R$ over $D$. In particular, slope ${ }_{D}(R)=2$ if $R$ is Koszul (Avramov, Conca and Iyengar [4]) and, when $D=S$ is a polynomial ring, $\operatorname{slope}_{S}(R) \leq a$ if the defining ideal of $R$ has a Gröbner basis of elements of degree $\leq a$. Similar results have been obtained by Park [24] under some restrictive assumptions. In the last section we discuss multigraded variants of the results presented.

\section{GENERAL BOUNDS}

In this section we consider a standard graded $K$-algebra $R$ with maximal homogeneous ideal $\mathfrak{m}$, which is a quotient of a polynomial ring $S$, say $R=S / I$ where $I$ is homogeneous 
(and may contain elements of degree 1). For a finitely generated graded $R$-module $M$ let $\beta_{i, j}^{R}(M)=\operatorname{dim}_{K} \operatorname{Tor}_{i}^{R}(M, K)_{j}$ be the graded Betti numbers of $M$ over $R$. We define the number

$$
t_{i}^{R}(M)=\max \left\{j \in \mathbb{Z}: \beta_{i, j}^{R}(M) \neq 0\right\},
$$

if $\operatorname{Tor}_{i}^{R}(M, K) \neq 0$ and $t_{i}^{R}(M)=-\infty$ otherwise. The (relative) regularity of $M$ over $R$ is given by

$$
\operatorname{reg}_{R}(M)=\sup \left\{t_{i}^{R}(M)-i: i \in \mathbb{N}\right\}
$$

and the Castelnuovo-Mumford regularity of $M$ is

$$
\operatorname{reg}(M)=\operatorname{reg}_{S}(M)=\sup \left\{t_{i}^{S}(M)-i: i \in \mathbb{N}\right\}
$$

it has also the cohomological interpretation

$$
\operatorname{reg}(M)=\max \left\{j: H_{\mathfrak{m}}^{i}(M)_{j-i} \neq 0 \text { for some } i \in \mathbb{N}\right\}
$$

where $H_{\mathfrak{m}}^{i}(M)$ denotes the $i$-th local cohomology module of $M$. One defines the slope of $M$ over $R$ by

$$
\operatorname{slope}_{R}(M)=\sup \left\{\frac{t_{i}^{R}(M)-t_{0}^{R}(M)}{i}: i \in \mathbb{N}, i>0\right\},
$$

and the Backelin rate of $R$ by

$$
\operatorname{Rate}(R)=\operatorname{slope}_{R}(\mathfrak{m})=\sup \left\{\frac{t_{i}^{R}(K)-1}{i-1}: i \in \mathbb{N}, i>1\right\} .
$$

The Backelin rate measures the deviation from being $\operatorname{Koszul}$ in general, $\operatorname{Rate}(R) \geq 1$, and $R$ is Koszul if and only if $\operatorname{Rate}(R)=1$. Finally, the Green-Lazarsfeld index of $R$ is given by

$$
\operatorname{index}(R)=\sup \left\{p \in \mathbb{N}: t_{i}^{S}(R) \leq i+1 \text { for every } i \leq p\right\} .
$$

It is the largest non-negative integer $p$ such that $R$ satisfies the property $N_{p}$. Note that we have index $(R)=\infty$ if and only if $\operatorname{reg}(R) \leq 1$, that is, the defining ideal of $R$ has a 2-linear resolution. On the other hand, index $(R) \geq 1$ if and only if $R$ is defined by quadrics. In general, $\operatorname{reg}(M)$ and $\operatorname{slope}_{R}(M)$ are finite (see [4]) while $\operatorname{reg}_{R}(M)$ can be infinite. However, $\operatorname{reg}_{R}(M)$ is finite if $R$ is Koszul, see Avramov and Eisenbud [5].

Remark 2.1. The invariants $\operatorname{reg}(M)$ and index $(R)$ are defined in terms of a presentation of $R$ as a quotient of a polynomial ring but do not depend on it. The assertion is a consequence of the following formula which is obtained, for example, from the graded analogue of [3, Theorem 2.2.3]: if $x \in R_{1}$ and $x M=0$, then

$$
\beta_{i, j}^{R}(M)=\beta_{i, j}^{R /(x)}(M)+\beta_{i-1, j-1}^{R /(x)}(M) .
$$

We record basic properties of these invariants. The modules are graded and finitely generated and the homomorphisms are of degree 0 .

Lemma 2.2. Let $R$ be a standard graded $K$-algebra, $N$ and $M_{j}, j \in \mathbb{N}$, be R-modules and $i \in \mathbb{N}$. 
(a) Let

$$
0 \rightarrow M_{1} \rightarrow M_{2} \rightarrow M_{3} \rightarrow 0
$$

be an exact sequence. Then

$$
\begin{aligned}
t_{i}^{R}\left(M_{1}\right) & \leq \max \left\{t_{i}^{R}\left(M_{2}\right), t_{i+1}^{R}\left(M_{3}\right)\right\}, \\
t_{i}^{R}\left(M_{2}\right) & \leq \max \left\{t_{i}^{R}\left(M_{1}\right), t_{i}^{R}\left(M_{3}\right)\right\}, \\
t_{i}^{R}\left(M_{3}\right) & \leq \max \left\{t_{i}^{R}\left(M_{2}\right), t_{i-1}^{R}\left(M_{1}\right)\right\} .
\end{aligned}
$$

(b) Let

$$
\cdots \rightarrow M_{k+1} \rightarrow M_{k} \rightarrow M_{k-1} \rightarrow \cdots \rightarrow M_{1} \rightarrow M_{0} \rightarrow N \rightarrow 0
$$

be an exact complex. Then

$$
t_{i}^{R}(N) \leq \max \left\{t_{i-j}^{R}\left(M_{j}\right): j=0, \ldots, i\right\}
$$

and

$$
\operatorname{reg}_{R}(N) \leq \sup \left\{\operatorname{reg}_{R}\left(M_{j}\right)-j: j \geq 0\right\} .
$$

(c) If $N$ vanishes in degree $>$ a then $t_{i}^{R}(N) \leq t_{i}^{R}(K)+a$.

(d) Let $J$ be a homogeneous ideal of $R$. If $\operatorname{reg}_{R}(R / J)=0$, then

$$
\operatorname{index}(R / J) \geq \operatorname{index}(R) \text {. }
$$

Proof. To prove (a) one just considers the long exact homology sequence for $\operatorname{Tor}^{R}(\cdot, K)$. For (b) one uses induction on $i$ and applies (a). Part (c) is proved by induction on $a-\min \left\{j: N_{j} \neq 0\right\}$. For (d) one applies (c) to the minimal free resolution of $R / J$ as an $R$-module. For every $i$ one gets $t_{i}^{S}(R / J) \leq \max \left\{t_{i-j}^{S}(R(-j)): j=0, \ldots i\right\}$. But we have $t_{i-j}^{S}(R(-j))=t_{i-j}^{S}(R)+j$. If $i \leq \operatorname{index}(R)$ then $t_{i-j}^{S}(R) \leq i-j+1$. It follows that $t_{i}^{S}(R / J) \leq i+1$ for every $i \leq \operatorname{index}(R)$. Hence index $(R / J) \geq \operatorname{index}(R)$.

Let $M$ be an $R$-module and let $K\left(\mathfrak{m}^{c}, M\right)$ be the graded Koszul complex associated to the $c$-th power of the maximal ideal of $R$. Let $Z_{i}\left(\mathfrak{m}^{c}, M\right), B_{i}\left(\mathfrak{m}^{c}, M\right), H_{i}\left(\mathfrak{m}^{c}, M\right)$ denote the $i$-th cycles, boundaries and homology of $K\left(\mathfrak{m}^{c}, M\right)$, respectively. We have:

Lemma 2.3. Set $Z_{i}=Z_{i}\left(\mathfrak{m}^{c}, M\right)$. For every $a \geq 0$ and $i \geq 0$ we have:

$$
\begin{aligned}
t_{a}^{R}\left(Z_{i+1}\right) \leq \max \{ & t_{a}^{R}(M)+(i+1) c, \\
& t_{a+1}^{R}\left(Z_{i}\right), \\
& \left.t_{0}^{R}\left(Z_{i}\right)+c+(a+1) \operatorname{Rate}(R)\right\} .
\end{aligned}
$$

Proof. Set $B_{i}=B_{i}\left(\mathfrak{m}^{c}, M\right)$ and $H_{i}=H_{i}\left(\mathfrak{m}^{c}, M\right)$. Recall that $\mathfrak{m}^{c} H_{i}=0$ and hence $H_{i}$ vanishes in degrees $>t_{0}\left(Z_{i}\right)+c-1$. It follows from Lemma 2.2(c) that

$$
t_{a}^{R}\left(H_{i}\right) \leq t_{0}^{R}\left(Z_{i}\right)+c-1+t_{a}^{R}(K) .
$$

The short exact sequences

$$
0 \rightarrow B_{i} \rightarrow Z_{i} \rightarrow H_{i} \rightarrow 0
$$

and

$$
0 \rightarrow Z_{i+1} \rightarrow K_{i+1} \rightarrow B_{i} \rightarrow 0
$$


now imply that

$$
\begin{aligned}
t_{a}^{R}\left(Z_{i+1}\right) & \leq \max \left\{t_{a}^{R}(M)+(i+1) c, t_{a+1}^{R}\left(B_{i}\right)\right\} \\
& \leq \max \left\{t_{a}^{R}(M)+(i+1) c, t_{a+1}^{R}\left(Z_{i}\right), t_{a+2}^{R}\left(H_{i}\right)\right\} \\
& \leq \max \left\{t_{a}^{R}(M)+(i+1) c, t_{a+1}^{R}\left(Z_{i}\right), t_{0}^{R}\left(Z_{i}\right)+c-1+t_{a+2}^{R}(K)\right\} .
\end{aligned}
$$

Since, by the very definition, $t_{a+2}^{R}(K) \leq 1+(a+1) \operatorname{Rate}(R)$ the desired result follows.

Lemma 2.3 allows us to bound $t_{a}^{R}\left(Z_{i}\right)$ inductively in terms of the various $t_{j}^{R}(M)$ and of $\operatorname{Rate}(R)$ :

Proposition 2.4. Set $Z_{i}=Z_{i}\left(\mathfrak{m}^{c}, M\right)$.

(a) Assume $c \geq \operatorname{slope}_{R}(M)$. Then for all $a, i \in \mathbb{N}$ we have

$$
t_{a}^{R}\left(Z_{i}\right) \leq t_{0}^{R}(M)+i c+\max \left\{a \text { slope }_{R}(M),(a+i) \operatorname{Rate}(R)\right\} .
$$

In particular, slope ${ }_{R}\left(Z_{i}\right) \leq \max \left\{\operatorname{slope}_{R} M,(1+i) \operatorname{Rate}(R)\right\}$.

(b) Assume $R$ is Koszul, i.e., Rate $(R)=1$. Then for all $a, i \in \mathbb{N}$ we have

$$
t_{a}^{R}\left(Z_{i}\right) \leq a+i(c+1)+\operatorname{reg}_{R}(M) .
$$

In particular, $\operatorname{reg}_{R}\left(Z_{i}\right) \leq i(c+1)+\operatorname{reg}_{R}(M)$.

Proof. To show (a) one uses that $t_{a}^{R}(M) \leq t_{0}^{R}(M)+a$ slope $_{R}(M)$ in combination with Lemma 2.3 and induction on $i$. For (b) one observes that $t_{a}^{R}(M) \leq a+\operatorname{reg}_{R}(M)$ in combination with Lemma 2.3 and induction on $i$.

In particular, we have the following corollary that generalizes Green's theorem [17, Theorem 2.2] to arbitrary standard graded $K$-algebras.

Corollary 2.5. Set $Z_{i}=Z_{i}\left(\mathfrak{m}^{c}, R\right)$. Then:

(a) $t_{0}^{R}\left(Z_{i}\right) \leq i c+\min \{i \operatorname{Rate}(R), i+\operatorname{reg}(R)\}$.

(b) $H_{i}\left(\mathfrak{m}^{c}, R\right)_{i c+j}=0$ for every $j \geq \min \{i \operatorname{Rate}(R), i+\operatorname{reg}(R)\}+c$.

Proof. To prove (a) one notes that setting $M=R$ and $a=0$ in Proposition 2.4(a) one has $t_{0}^{R}\left(Z_{i}\right) \leq i c+i \operatorname{Rate}(R)$. Then one considers $R$ as an $S$-module and sets $M=R$ and $a=0$ in Proposition $2.4(\mathrm{~b})$. One has $t_{0}^{S}\left(Z_{i}\right) \leq i(c+1)+\operatorname{reg}(R)$. Since $t_{0}^{S}\left(Z_{i}\right)=t_{0}^{R}\left(Z_{i}\right)$ we are done. To prove (b) one uses (a) and the fact that $\mathfrak{m}^{c} H_{i}\left(\mathfrak{m}^{c}, R\right)=0$.

\section{KOSZUL CYCLES}

In this section we concentrate our attention on the Koszul complex $K\left(\mathfrak{m}^{c}\right)=K\left(\mathfrak{m}^{c}, S\right)$ where $S=K\left[X_{1}, \ldots, X_{n}\right]$ is a standard graded polynomial ring over a field $K$ and $\mathfrak{m}=$ $\left(X_{1}, \ldots, X_{n}\right)$ is its maximal homogeneous ideal. The Koszul complex $K\left(\mathfrak{m}^{c}\right)$ is indeed an $S$-algebra, namely the exterior algebra $S \otimes_{K} \bigwedge^{\bullet} S_{c} \cong \Lambda^{\bullet} F$ where $F$ is the free $S$-module of rank equal to $\operatorname{dim}_{K} S_{c}=\left(\begin{array}{c}n-1+c \\ n-1\end{array}\right)$. The differential of $K\left(\mathfrak{m}^{c}\right)$ is denoted by $\partial$; it is an antiderivation of degree -1 . We consider the cycles $Z_{t}\left(\mathfrak{m}^{c}, S\right)$, simply denoted by $Z_{t}\left(\mathfrak{m}^{c}\right)$, of the Koszul complex $K\left(\mathfrak{m}^{c}\right)$, and the $S$-subalgebra $Z\left(\mathfrak{m}^{c}\right)=\bigoplus_{t} Z_{t}\left(\mathfrak{m}^{c}\right)$ of $K\left(\mathfrak{m}^{c}\right)$.

For $f_{1}, \ldots, f_{t} \in S_{c}$ and $g \in S$ we set

$$
g\left[f_{1}, \ldots, f_{t}\right]=g \otimes f_{1} \wedge \cdots \wedge f_{t} \in K_{t}\left(\mathfrak{m}^{c}\right) .
$$


The elements $\left[u_{1}, \ldots, u_{t}\right]$ for distinct monomials $u_{1}, u_{2}, \ldots, u_{t}$ of degree $c$ (ordered in some way) form a basis of $K_{t}\left(\mathfrak{m}^{c}\right)$ as an $S$-module. We call them monomial free generators of $K_{t}\left(\mathfrak{m}^{c}\right)$. The elements $v\left[u_{1}, \ldots, u_{t}\right]$, where $u_{1}, u_{2}, \ldots, u_{t}$ are distinct monomials of degree $c$ and $v$ is a monomial of arbitrary degree, form a basis of the $K$-vector space $K_{t}\left(\mathfrak{m}^{c}\right)$. They are called monomials of $K_{t}\left(\mathfrak{m}^{c}\right)$. Evidently $K\left(\mathfrak{m}^{c}\right)$ is a $\mathbb{Z}$-graded complex, but it is also $\mathbb{Z}^{n}$-graded with the following assignment of degrees: $\operatorname{deg} v\left[u_{1}, \ldots, u_{t}\right]=\alpha$ where $v u_{1} \cdots u_{t}=X^{\alpha}$.

Every element $z \in K_{t}\left(\mathfrak{m}^{c}\right)$ can be written uniquely as a linear combination

$$
z=\sum f_{i}\left[u_{i 1}, \ldots, u_{i t}\right]
$$

of monomial free generators $\left[u_{i 1}, \ldots, u_{i t}\right]$ with coefficients $f_{i} \in S$. We call $f_{i}$ the coefficient of $\left[u_{i 1}, \ldots, u_{i t}\right]$ in $z$. Note that $z$ is $\mathbb{Z}$-homogenous of degree $c t+j$ if every $f_{i}$ is homogeneous of degree $j$. In this case $z$ has coefficients of degree $j$. Note also that $z$ is homogeneous of degree $\alpha \in \mathbb{Z}^{n}$ in the $\mathbb{Z}^{n}$-grading if for every $i$ one has $f_{i}=\lambda_{i} v_{i}$ such that $\lambda_{i} \in K$ and $v_{i}$ is a monomial with $v_{i} u_{i 1} \cdots u_{i t}=X^{\alpha}$. Given $z \in K\left(\mathfrak{m}^{c}\right)$ and a monomial $v\left[u_{1}, \ldots, u_{t}\right]$ we say that $v\left[u_{1}, \ldots, u_{t}\right]$ appears in $z$ if it has a non-zero coefficient in the representation of $z$ as $K$-linear combination of monomials of $K\left(\mathfrak{m}^{c}\right)$. An immediate consequence of Proposition 2.4 is:

Lemma 3.1. We have $\operatorname{reg}\left(Z_{t}\left(\mathfrak{m}^{c}\right)\right) \leq t(c+1)$. In particular, $Z_{t}\left(\mathfrak{m}^{c}\right)$ is generated by elements of degree $\leq t(c+1)$.

Remark 3.2. It is easy to see and well known that $Z_{1}\left(\mathfrak{m}^{c}\right)$ is generated by the elements $X_{i}\left[X_{j} b\right]-X_{j}\left[X_{i} b\right]$ where $b$ is a monomial of degree $c-1$.

We write $Z_{1}\left(\mathfrak{m}^{c}\right)^{t}$ for $\bigwedge^{t} Z_{1}\left(\mathfrak{m}^{c}\right) \subset Z_{t}\left(\mathfrak{m}^{c}\right)$, and similarly for other products.

Theorem 3.3. For every t the module $Z_{t}\left(\mathfrak{m}^{c}\right) / Z_{1}\left(\mathfrak{m}^{c}\right)^{t}$ is generated in degree $<t(c+1)$.

Proof. The assertion is proved by induction on $t$. For $t=1$ there is nothing to do. By induction it is enough to verify that $Z_{t}\left(\mathfrak{m}^{c}\right) / Z_{1}\left(\mathfrak{m}^{c}\right) Z_{t-1}\left(\mathfrak{m}^{c}\right)$ is generated in degree < $t(c+1)$. Since $Z_{t}\left(\mathfrak{m}^{c}\right)$ is $\mathbb{Z}^{n}$-graded and generated in degree $\leq t(c+1)$, it suffices to show that every $\mathbb{Z}^{n}$-graded element $f \in Z_{t}\left(\mathfrak{m}^{c}\right)$ of total degree $t(c+1)$ can be written modulo $Z_{1}\left(\mathfrak{m}^{c}\right) Z_{t-1}\left(\mathfrak{m}^{c}\right)$ as a multiple of an element in $Z_{t}\left(\mathfrak{m}^{c}\right)$ of total degree $<t(c+1)$. Let $\alpha \in \mathbb{Z}^{n}$ be the $\mathbb{Z}^{n}$-degree of $f$. Permuting the coordinates if necessary, we may assume $\alpha_{n}>0$.

Let $u \in S$ be a monomial of degree $c$ with $X_{n} \mid u$. We write $f=a+b[u]$ with $a \in K_{t}\left(\mathfrak{m}^{c}\right)$ and $b \in K_{t-1}\left(\mathfrak{m}^{c}\right)$ such that $a, b$ involve only free generators $\left[u_{1}, \ldots, u_{s}\right](s=t, t-1)$ with $u_{i} \neq u$ for all $i$. Since

$$
0=\partial(f)=\partial(a)+\partial(b)[u] \pm b u
$$

it follows that $\partial(b)=0$, i.e., $b \in Z_{t-1}\left(\mathfrak{m}^{c}\right)$. Note that $b$ has coefficients of degree $t$. Since $Z_{t-1}\left(\mathfrak{m}^{c}\right)$ is generated by elements with coefficients of degree $\leq t-1$ we may write

$$
b=\sum_{j=1}^{s} \lambda_{j} v_{j} z_{j}
$$

where $\lambda_{j} \in K, z_{j} \in Z_{t-1}\left(\mathfrak{m}^{c}\right)$ and the $v_{j}$ are monomials of positive degree. 
Let $\lambda_{j} v_{j} z_{j}$ be a summand in (2). If $X_{n}$ does not divide $v_{j}$, then choose $i<n$ such that $X_{i} \mid v_{j}$. We set $z^{\prime}=X_{i}[u]-X_{n}\left[u^{\prime}\right] \in Z_{1}\left(\mathfrak{m}^{c}\right)$ where $u^{\prime}=u X_{i} / X_{n}$, and subtract from $f$ the element

$$
\lambda_{j} \frac{v_{j}}{X_{i}} z_{j} z^{\prime} \in Z_{t-1}\left(\mathfrak{m}^{c}\right) Z_{1}\left(\mathfrak{m}^{c}\right)
$$

Repeating this procedure for each $\lambda_{j} v_{j} z_{j}$ in (2) such that $X_{n}$ does not divide $v_{j}$ we obtain a cycle $f_{1} \in Z_{t}\left(\mathfrak{m}^{c}\right)$ of degree $\alpha$ such that

(i) $f=f_{1} \bmod Z_{1}\left(\mathfrak{m}^{c}\right) Z_{t-1}\left(\mathfrak{m}^{c}\right)$;

(ii) if a monomial $v\left[u_{1}, \ldots, u_{t}\right]$ appears in $f_{1}$ and $u \in\left\{u_{1}, \ldots, u_{t}\right\}$, then $X_{n} \mid v$.

We repeat the described procedure for each monomial $u$ of degree $c$ with $X_{n} \mid u$. We end up with an element $f_{2} \in Z_{t}\left(\mathfrak{m}^{c}\right)$ of degree $\alpha$ such that

(iii) $f=f_{2} \bmod Z_{1}\left(\mathfrak{m}^{c}\right) Z_{t-1}\left(\mathfrak{m}^{c}\right)$;

(iv) if a monomial $v\left[u_{1}, \ldots, u_{t}\right]$ appears in $f_{2}$ and $X_{n} \mid u_{1} \cdots u_{t}$, then $X_{n} \mid v$.

Note that if $v\left[u_{1}, \ldots, u_{t}\right]$ appears in $f_{2}$ and $X_{n} \nmid u_{1} \cdots u_{t}$, then $X_{n} \mid v$ by degree reasons. Hence for every monomial $v\left[u_{1}, \ldots, u_{t}\right]$ appearing in $f_{2}$ we have $X_{n} \mid v$. Therefore $f_{2}=X_{n} g$, and $g \in Z_{t}\left(\mathfrak{m}^{c}\right)$ has degree $<t(c+1)$. This completes the proof.

Next we describe some cycles which are needed in the following. For $t \in \mathbb{N}, t \geq 1$ let $\mathscr{S}_{t}$ be the group of permutations of $\{1, \ldots, t\}$.

Lemma 3.4. Let $s, t$ be integers such that $1 \leq s \leq c$ and $t>0$. Let $a_{1}, a_{2} \ldots, a_{t+1} \in S$ be monomials of degree $s$ and $b_{1}, b_{2} \ldots, b_{t} \in S$ monomials of degree $c-s$. Then

$$
\sum_{\sigma \in \mathscr{S}_{t+1}}(-1)^{\sigma} a_{\sigma(t+1)}\left[b_{1} a_{\sigma(1)}, b_{2} a_{\sigma(2)}, \ldots, b_{t} a_{\sigma(t)}\right]
$$

belongs to $Z_{t}\left(\mathfrak{m}^{c}\right)$.

Proof. We apply the differential of $K\left(\mathfrak{m}^{c}\right)$ to (3) and observe that for distinct integers $j_{1}, j_{2}, \ldots, j_{i-1}, j_{i+1}, \ldots, j_{t}$ in the range of 1 to $t+1$ the free generator

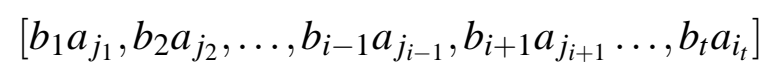

appears twice in the image. The coefficients differ just by -1 because the corresponding permutations differ by a transposition. Thus the element in (3) is indeed a cycle.

\section{Remark 3.5.}

(a) Of course, it may happen that a cycle described in Lemma 3.4 is identically 0. But for $t=1$ and $s=1$ these cycles take the form

$$
X_{i}\left[b X_{j}\right]-X_{j}\left[b X_{i}\right]
$$

and, as said already in Remark 3.2, they generate $Z_{1}\left(\mathfrak{m}^{c}\right)$. For $s=c$ the cycles in Lemma 3.4 are the boundaries of $K_{t}\left(\mathfrak{m}^{c}\right)$ (multiplied by $t$ !). Hence for $c=1$ the cycles in 3.4 generate the algebra $Z(\mathfrak{m})$. So there is some evidence that the cycles in Lemma 3.4 might generate $Z\left(\mathfrak{m}^{c}\right)$ in general. 
(b) For $n=3, c=2, t=2$ and $s=1$ with $a_{i}=X_{i}$ for $i=1,2,3$ and $b_{i}=X_{i}$ for $i=1,2$ the cycle in (3) is

$$
\begin{array}{cccccc} 
& & & & 0 \\
+ & X_{3}\left[X_{1}^{2}, X_{2}^{2}\right] & - & X_{2}\left[X_{1}^{2}, X_{2} X_{3}\right] & - & \overbrace{3}\left[X_{1} X_{2}, X_{1} X_{2}\right] \\
+ & X_{1}\left[X_{1} X_{2}, X_{2} X_{3}\right] & + & X_{2}\left[X_{1} X_{3}, X_{1} X_{2}\right] & - & X_{1}\left[X_{1} X_{3}, X_{2}^{2}\right],
\end{array}
$$

a non-zero element in $Z_{2}\left(\mathfrak{m}^{2}\right)$.

Let $B_{i}\left(\mathfrak{m}^{c}\right) \subset Z_{i}\left(\mathfrak{m}^{c}\right)$ denote the $S$-module of boundaries in $K_{i}\left(\mathfrak{m}^{c}\right)$.

Theorem 3.6. We have

$$
(c+1) ! \mathfrak{m}^{c-1} Z_{1}\left(\mathfrak{m}^{c}\right)^{c} \subset B_{c}\left(\mathfrak{m}^{c}\right) .
$$

Proof. For a monomial $b \in S$ of degree $c-1$ and variables $X_{i}, X_{j}$ we set

$$
z_{b}\left(X_{i}, X_{j}\right)=X_{i}\left[b X_{j}\right]-X_{j}\left[b X_{i}\right]
$$

As observed in Remark 3.2, the elements $z_{b}\left(X_{i}, X_{j}\right)$ generate $Z_{1}\left(\mathfrak{m}^{c}\right)$. Let $a, b \in S$ be monomials of degree $c-1$. We note that

$$
a z_{b}\left(X_{i}, X_{j}\right)+b z_{a}\left(X_{i}, X_{j}\right)=\partial\left(\left[a X_{i}, b X_{j}\right]+\left[b X_{i}, a X_{j}\right]\right) \in B_{1}\left(\mathfrak{m}^{c}\right),
$$

that is,

$$
a z_{b}\left(X_{i}, X_{j}\right)=-b z_{a}\left(X_{i}, X_{j}\right) \quad \bmod B_{1}\left(\mathfrak{m}^{c}\right) .
$$

Let $b_{1}, \ldots, b_{c+1} \in S$ be monomials of degree $c-1$, and let $X_{i j} \in\left\{X_{1}, \ldots, X_{n}\right\}$ for $i=$ $1, \ldots, c$ and $j=0,1$ be variables. By construction, the elements

$$
f=b_{c+1} \prod_{i=1}^{c} z_{b_{i}}\left(X_{i 0}, X_{i 1}\right) \in Z_{c}\left(\mathfrak{m}^{c}\right)
$$

generate $\mathfrak{m}^{c-1} Z_{1}\left(\mathfrak{m}^{c}\right)^{c}$. We have to show that $(c+1) ! f \in B_{c}\left(\mathfrak{m}^{c}\right)$.

Let $\sigma \in \mathscr{S}_{c+1}$ be an arbitrary permutation. From Equation (4) and from the inclusion $B_{1}\left(\mathfrak{m}^{c}\right) Z_{c-1}\left(\mathfrak{m}^{c}\right) \subset B_{c}\left(\mathfrak{m}^{c}\right)$ it follows that

$$
f=(-1)^{\sigma} b_{\sigma(c+1)} \prod_{i=1}^{c} z_{b_{\sigma(i)}}\left(X_{i 0}, X_{i 1}\right) \quad \bmod B_{c}\left(\mathfrak{m}^{c}\right) .
$$

Hence

$$
(c+1) ! f=\sum_{\sigma \in \mathscr{S}_{c+1}}(-1)^{\sigma} b_{\sigma(c+1)} \prod_{i=1}^{c} z_{b_{\sigma(i)}}\left(X_{i 0}, X_{i 1}\right) \quad \bmod B_{c}\left(\mathfrak{m}^{c}\right) .
$$

In the right-hand side of (5) we replace $z_{b_{\sigma(i)}}\left(X_{i 0}, X_{i 1}\right)$ with $X_{i 0}\left[b_{\sigma(i)} X_{i 1}\right]-X_{i 1}\left[b_{\sigma(i)} X_{i 0}\right]$, then expand the product and collect the multiples of $X_{1 j_{1}} \cdots X_{c j_{c}}$ for $j=\left(j_{1}, \ldots, j_{c}\right) \in$ $\{0,1\}^{c}$. We can rewrite Equation (5) as

$$
(c+1) ! f=\sum_{j \in\{0,1\}^{c}}(-1)^{j_{1}+\cdots+j_{c}} X_{1 j_{1}} \cdots X_{c j_{c}} W_{j} \quad \bmod B_{c}\left(\mathfrak{m}^{c}\right),
$$

where

$$
W_{j}=\sum_{\sigma \in \mathscr{S}_{c+1}}(-1)^{\sigma} b_{\sigma(c+1)}\left[X_{1 i_{1}} b_{\sigma(1)}, \ldots, X_{c i_{c}} b_{\sigma(c)}\right]
$$


with $i_{k}=1-j_{k}$ for $k=1, \ldots, c$. Lemma 3.4 yields $W_{j} \in Z_{c}\left(\mathfrak{m}^{c}\right)$. Since $\mathfrak{m}^{c} Z_{c}\left(\mathfrak{m}^{c}\right) \subset$ $B_{c}\left(\mathfrak{m}^{c}\right)$ we get

$$
X_{1 j_{1}} \cdots X_{c j_{c}} W_{j}=0 \quad \bmod B_{c}\left(\mathfrak{m}^{c}\right) .
$$

Thus Equation (6) implies $(c+1) ! f \in B_{c}\left(\mathfrak{m}^{c}\right)$ as desired.

As a consequence we obtain:

Corollary 3.7. The homology $H_{t}\left(\mathfrak{m}^{c}\right)_{t c+j}$ vanishes if $j \geq t+c$. If $t \geq c$ and the characteristic of $K$ is either 0 or $>c+1$, then $H_{t}\left(\mathfrak{m}^{c}\right)_{t c+j}=0$ for $j \geq t+c-1$.

Proof. The first statement is a special case of Corollary 2.5. For the second, set $j=$ $t+c-1$. We have to prove that $H_{t}\left(\mathfrak{m}^{c}\right)_{t c+j}=0$. Theorem 3.3 implies that $Z_{t}\left(\mathfrak{m}^{c}\right)$ is generated by some elements $z_{i}$ of degree $<t(c+1)$ and by some elements $w_{i}$ of $Z_{1}\left(\mathfrak{m}^{c}\right)^{t}$ of degree $t(c+1)$. Hence an element $f \in Z_{t}\left(\mathfrak{m}^{c}\right)_{t c+j}$ can be written as $f=\sum a_{i} z_{i}+\sum b_{i} w_{i}$ with $a_{i} \in \mathfrak{m}^{c}$ and $b_{i} \in \mathfrak{m}^{c-1}$ by degree reasons. Now $\sum a_{i} z_{i} \in \mathfrak{m}^{c} Z_{t}\left(\mathfrak{m}^{c}\right) \subset B_{t}\left(\mathfrak{m}^{c}\right)$. In view of Theorem 3.6 we furthermore have

$$
\sum b_{i} w_{i} \in \mathfrak{m}^{c-1} Z_{1}\left(\mathfrak{m}^{c}\right)^{t}=\mathfrak{m}^{c-1} Z_{1}\left(\mathfrak{m}^{c}\right)^{c} Z_{1}\left(\mathfrak{m}^{c}\right)^{t-c} \subset B_{c}\left(\mathfrak{m}^{c}\right) Z_{1}\left(\mathfrak{m}^{c}\right)^{t-c} \subset B_{t}\left(\mathfrak{m}^{c}\right) .
$$

Summing up, $f \in B_{t}\left(\mathfrak{m}^{c}\right)$ and hence $H_{t}\left(\mathfrak{m}^{c}\right)_{t c+j}=0$.

Remark 3.8. The coefficient $(c+1)$ ! in Theorem 3.6 and the assumption on the characteristic in Corollary 3.7 are necessary. For $n=7, c=2$ and char $K=3$ we have checked that $\mathfrak{m} Z_{1}\left(\mathfrak{m}^{2}\right)^{2} \not \subset B_{2}\left(\mathfrak{m}^{2}\right)$ and that $\operatorname{dim} H_{2}\left(\mathfrak{m}^{2}\right)_{7}=1$. More precisely, $H_{2}\left(\mathfrak{m}^{2}\right)$ has dimension 1 in the multidegree $(1,1,1,1,1,1,1)$ if $\operatorname{char} K=3$.

Another consequence of Theorem 3.6 is the following:

Corollary 3.9. Assume char $K$ is 0 or $>c+1$. Then $\operatorname{reg} Z_{t+1}\left(\mathfrak{m}^{c}\right) \leq(t+1)(c+1)-1$ for every $t \geq c$. In particular, $Z_{1}\left(\mathfrak{m}^{c}\right)^{c+1} \subset \mathfrak{m} Z_{c+1}\left(\mathfrak{m}^{c}\right)$.

Proof. To prove the first assertion, let us denote by $Z_{t}$ the module $Z_{t}\left(\mathfrak{m}^{c}\right)$ and similarly for $B_{t}, H_{t}$ and $K_{t}$. The short exact sequences

$$
0 \rightarrow B_{t} \rightarrow Z_{t} \rightarrow H_{t} \rightarrow 0 \text { and } 0 \rightarrow Z_{t+1} \rightarrow K_{t+1} \rightarrow B_{t} \rightarrow 0
$$

imply that $\operatorname{reg}\left(Z_{t+1}\right) \leq \max \left\{\operatorname{reg}\left(Z_{t}\right)+1, \operatorname{reg}\left(H_{t}\right)+2\right\}$. Using Lemma 3.1 and Corollary 3.7 one obtains $\operatorname{reg}\left(Z_{t+1}\right) \leq(t+1)(c+1)-1$ for every $t \geq c$. The second assertion follows immediately from the first.

\section{THE GREEN-LAZARSFELD INDEX OF VERONESE SUBRINGS OF POLYNOMIAL RINGS}

Again we consider a standard graded $K$-algebra $R$ of the form $R=S / I$ where $K$ is a field, $S=K\left[X_{1}, \ldots, X_{n}\right]$ is a polynomial ring over $K$ graded by $\operatorname{deg}\left(X_{i}\right)=1$ and $I \subset S$ is a graded ideal.

Given $c \in \mathbb{N}, c \geq 1$ and $0 \leq k<c$, we set

$$
V_{R}(c, k)=\bigoplus_{i \in \mathbb{N}} R_{k+i c}
$$


Observe that $R^{(c)}=V_{R}(c, 0)$ is the usual $c$-th Veronese subring of $R$, and that the $V_{R}(c, k)$ are $R^{(c)}$-modules known as the Veronese modules of $R$. For a finitely generated graded $R$-module $M$ we similarly define

$$
M^{(c)}=\bigoplus_{i \in \mathbb{Z}} M_{i c}
$$

We consider $R^{(c)}$ as a standard graded $K$-algebra with homogeneous component of degree $i$ equal to $R_{i c}$, and $M^{(c)}$ as a graded $R^{(c)}$-module with homogeneous components $M_{i c}$. The grading of the $R^{(c)}$-module $V_{R}(c, k)$ is given by $V_{R}(c, k)_{i}=R_{k+i c}$. In particular, the latter modules are all generated in degree 0 with respect to this grading.

Let $T=\operatorname{Sym}\left(R_{c}\right)$ be the symmetric algebra on vector space $R_{c}$, that is,

$$
T=K\left[Y_{u}: u \in B_{c}\right]
$$

where $B_{c}$ is any $K$-basis of $R_{c}$. When $R=S$ the basis $B_{c}$ can be taken as the set of monomials of degree $c$. The canonical map $T \rightarrow R^{(c)}$ is surjective, and the modules $V_{R}(c, k)$ are also finitely generated graded $T$-modules (generated in degree 0 ).

With the notation of the preceding sections we have:

Lemma 4.1. For $i \in \mathbb{N}, j \in \mathbb{Z}$ and $0 \leq k<c$ we have

$$
\beta_{i, j}^{T}\left(V_{R}(c, k)\right)=\operatorname{dim}_{K} H_{i}\left(\mathfrak{m}^{c}, R\right)_{j c+k} .
$$

Proof. Let $K\left(T_{1}\right)$ be the Koszul complex (of $T$-modules) associated to the elements $Y_{u}$ with $u \in B_{c}$. We observe that

$$
\left.\beta_{i, j}^{T}\left(V_{R}(c, k)\right)=\operatorname{Tor}_{i}^{T}\left(K, V_{R}(c, k)\right)_{j}=\operatorname{dim}_{K} H_{i}\left(K\left(T_{1}\right)\right) \otimes_{T} V_{R}(c, k)\right)_{j} .
$$

But the last homology is $H_{i}\left(\mathfrak{m}^{c}\right)_{j c+k}$, the $i$-th homology of the complex $K\left(\mathfrak{m}^{c}\right)_{j c+k}$.

Lemma 4.1 and Corollary 3.7 imply:

Corollary 4.2. For all integers $i \geq 0$ and $k=0, \ldots, c-1$ we have

$$
t_{i}^{T}\left(V_{S}(c, k)\right)<1+i+\frac{i-k}{c} .
$$

If $K$ has characteristic 0 or $>c+1$ and $i \geq c$, then

$$
t_{i}^{T}\left(V_{S}(c, k)\right)<1+i+\frac{i-k-1}{c} .
$$

Remark 4.3. Let $S=K\left[X_{1}, \ldots, X_{n}\right]$. Andersen [1] proved that the graded Betti numbers $\beta_{i j}^{T}\left(S^{(2)}\right)$ do not depend on the characteristic of $K$ if $i \leq 4$ or if $i=5$ and $n \leq 6$. She also proved that, for $n \geq 7$, one has $\beta_{5,7}^{T}\left(S^{(2)}\right) \neq 0$ in characteristic 5 while $\beta_{5,7}^{T}\left(S^{(2)}\right)=0$ in characteristic 0 . Thus, for $n \geq 7$ one has

$$
\operatorname{index}\left(S^{(2)}\right)= \begin{cases}5, & \operatorname{char} K=0 \\ 4, & \operatorname{char} K=5\end{cases}
$$

Also note that already $\beta_{2,3}\left(V_{S}(2,1)\right)$ depends on the characteristic if $n \geq 7$, as follows from the data in Remark 3.8 . 
We now record a duality on $H\left(\mathfrak{m}^{c}\right)$. It can be seen as an Avramov-Golod type duality (see [8, Theorem 3.4.5]) or as an algebraic version of Serre duality.

Proposition 4.4. Let $N=\left(\begin{array}{c}n+c-1 \\ c\end{array}\right)$. Then

$$
\operatorname{dim}_{K} H_{i}\left(\mathfrak{m}^{c}\right)_{j}=\operatorname{dim}_{K} H_{N-n-i}\left(\mathfrak{m}^{c}\right)_{N c-n-j}, \quad i, j \in \mathbb{Z}, i, j \geq 0 .
$$

Proof. For this proof (and only here) we consider the grading on the polynomial ring $T=K\left[Y_{u}: u \in S\right.$ monomial, $\left.\operatorname{deg} u=c\right]$ in which $Y_{u}$ has degree $c$. The polynomial ring $S$ in its standard grading is a finitely generated graded $T$-module as usual.

Note that the canonical module of $S$ is $\omega_{S}=S(-n)$, and that the canonical module of $T$ is $\omega_{T}=T(-N c)$. Recall that

$$
\operatorname{Ext}_{T}^{j}(S, T(-N c))= \begin{cases}0 & \text { if } j<N-n \\ S(-n) & \text { if } j=N-n .\end{cases}
$$

(See, e.g., [8, Theorem 3.3.7 and Theorem 3.3.10].) Let $F$ be a minimal graded free $T$ resolution of $S$. Computing $\operatorname{Ext}_{T}^{i}(S, T(-N c))$ via $\operatorname{Hom}_{T}(F, T(-N c))$, the minimal graded free $T$-resolution of $S(-n)$, we see immediately that $\beta_{i, j}^{T}(S)=\beta_{N-n-i, N c-j}^{T}(S(-n))$. Then

$$
\operatorname{dim}_{K} H_{i}\left(\mathfrak{m}^{c}\right)_{j}=\beta_{i, j}^{T}(S)=\beta_{N-n-i, N c-n-j}^{T}(S)=\operatorname{dim}_{K} H_{N-n-i}\left(\mathfrak{m}^{c}\right)_{N c-n-j} .
$$

Example 4.5. Let char $K=0$. Computer algebra systems as CoCoA [10], Macaulay 2 [16] or Singular [20] can easily compute the following diagram for $\operatorname{dim}_{K} H\left(\mathfrak{m}^{3}\right)$ in the case $n=3$ :

\begin{tabular}{c|ccccccccc} 
& 0 & 1 & 2 & 3 & 4 & 5 & 6 & 7 & \\
\hline 0 & 1 & - & - & - & - & - & - & - & $\leftarrow$ \\
1 & 3 & 15 & 21 & - & - & - & - & - & \\
2 & 6 & 49 & 105 & 147 & 105 & 21 & - & - & \\
3 & $\mathbf{0}$ & 27 & 105 & 189 & 189 & 105 & 27 & - & $\leftarrow$ \\
4 & - & $\mathbf{0}$ & 21 & 105 & 147 & 105 & 49 & 6 & \\
5 & - & - & $\mathbf{0}$ & 0 & - & 21 & 15 & 3 & \\
6 & - & - & - & $\mathbf{0}$ & 0 & - & - & 1 & $\leftarrow$
\end{tabular}

The $(i, j)$-entry of the table is $\operatorname{dim}_{K} H_{i}\left(\mathfrak{m}^{c}\right)_{i c+j}$ and - indicates that entry is 0 . By selecting the rows whose indices are multiples of $c=3$ (those marked by the arrows in the diagram) one gets the Betti diagram of $S^{(3)}$. Green's theorem [17. Thm.2.2] implies the vanishing in the positions of the boldface zeros and below. Our result implies the vanishing in the positions of the non-bold zeros and below. (Also see Weyman [29, Example 7.2.7] for the case $n=c=3$.)

Using the duality we prove the upper bound for index $\left(S^{(c)}\right)$ due to Ottaviani and Paoletti [23] (in arbitrary characteristic). To this end we need a variation of [25, Corollary 2.10].

Proposition 4.6. Let $\left(e_{i}: i=1, \ldots, m\right)$ be a basis of the vector space $\bigwedge^{t} S_{c}$ (thus $m=\left(\begin{array}{c}N \\ t\end{array}\right)$ with $\left.N=\left(\begin{array}{c}n-1+c \\ n-1\end{array}\right)\right)$. Let

$$
z=\sum_{i=1}^{m} f_{i} e_{i}
$$

be a non-zero element in $Z_{t}\left(\mathfrak{m}^{c}\right)$. Then the $K$-vector space generated by the coefficients $f_{i}$ of $z$ has dimension $\geq t+1$. 
Proof. Since the $K$-vector space dimension of the space of coefficients does not depend on the basis, it is enough to prove the assertion for the monomial basis $\left(e_{i}\right)$. We use induction on $t$.

The case $t=0$ is obvious. So assume $t>0$. Fix a term order, for example the lexicographic term order, on $S$. Let $C(z)$ denote the vector space generated by the coefficients of $z$. As already discussed in the proof of Theorem 3.3. for every monomial $u$ of degree $c$ we may write $z=a+b[u]$ with $b \in Z_{t-1}\left(\mathfrak{m}^{c}\right)$. Choose $u$ to be the largest monomial (with respect to the term order) such that the corresponding $b$ is non-zero. By induction $\operatorname{dim}_{K} C(b) \geq t$ and $C(b) \subset C(z)$.

If $C(b) \neq C(z)$ then clearly $\operatorname{dim}_{K} C(z) \geq t+1$. If instead $C(b)=C(z)$, then $C(a) \subset$ $C(b)$. Let $v$ be the largest monomial appearing in the elements of $C(b)$. The inclusion $C(a) \subset C(b)$ implies that every monomial appearing in the elements of $C(a)$ is $\leq v$. But $\partial(a) \pm b u=0$ and hence $C(\partial(a))=C(b u)=u C(b)$. The monomial $v u$ appears in $C(b u)$. Every monomial in $C(\partial(a))$ is of the form $w u_{1}$ where $w$ is a monomial appearing in $C(a)$ and $u_{1}$ is a monomial of degree $c$ which is an "exterior" factor of some free generator appearing in $z$. By construction $w \leq v$ and $u_{1}<u$. It follows that $w u_{1} \neq v u$, a contradiction with $C(\partial(a))=u C(b)$.

Theorem 4.7. For $n \geq 3$ and $c \geq 3$ one has index $\left(S^{(c)}\right) \leq 3 c-3$, and equality holds for $n=3$.

Proof. We first consider the case $n=3$. By an inspection of the Hilbert function of (the Cohen-Macaulay ring) $S^{(c)}$ one sees immediately that $\operatorname{reg} S^{(c)} \leq 2$, that is, $t_{i}^{T}\left(S^{(c)}\right) \leq i+2$ for every $i \geq 0$. From Theorem 4.1 and Proposition 4.4 we have

$$
\beta_{i, j}^{T}\left(S^{(c)}\right)=\operatorname{dim}_{K} H_{i}\left(\mathfrak{m}^{c}\right)_{j c}=\operatorname{dim}_{K} H_{N-3-i}\left(\mathfrak{m}^{c}\right)_{N c-3-j c} .
$$

Therefore $t_{i}^{T}\left(S^{(c)}\right) \leq i+1$ if and only if

$$
H_{N-3-i}\left(\mathfrak{m}^{c}\right)_{(N-3-i) c+c-3}=0
$$

and, since the boundaries have coefficients of degree $\geq c$, this is equivalent to

$$
Z_{N-3-i}\left(\mathfrak{m}^{c}\right)_{(N-3-i) c+c-3}=0 \text {. }
$$

So we have to analyze the cycles in $Z_{N-3-i}\left(\mathfrak{m}^{c}\right)$ with coefficients of degree $c-3$.

It follows from Proposition 4.6 that

$$
N-3-i+1 \leq \operatorname{dim}_{K} S_{c-3}=\left(\begin{array}{c}
c-1 \\
2
\end{array}\right)
$$

if there exists a non-zero cycle $z \in Z_{N-3-i}\left(\mathfrak{m}^{c}\right)$ with coefficients of degree $c-3$. Thus there are no cycles in that degree if $N-3-i \geq\left(\begin{array}{c}c-1 \\ 2\end{array}\right)$. Hence

$$
t_{i}^{T}\left(S^{(c)}\right) \leq i+1 \text { for } 0 \leq i \leq 3 c-3,
$$

that is, index $\left(S^{(c)}\right) \geq 3 c-3$. It remains to show that $S^{(c)}$ does not satisfy the property $N_{3 c-2}$. We have to find a non-zero cycle in $Z_{j}\left(\mathfrak{m}^{c}\right)$ with coefficients of degree $c-3$ where $j=N-3-i$. Note that $j=\left(\begin{array}{c}c-1 \\ 2\end{array}\right)-1$ and so $j+1=\operatorname{dim} S_{c-3}$. Take the monomials $u_{1}^{\prime}, \ldots, u_{j+1}^{\prime}$ of degree $c-3$ and set $u_{k}=u_{k}^{\prime} X_{1} X_{2} X_{3}$ for $k=1, \ldots, j+1$. Then

$$
w=\partial\left(\left[u_{1}, \ldots, u_{j+1}\right]\right) \in Z_{j}\left(\mathfrak{m}^{c}\right)
$$


is non-zero boundary with coefficients of degree $c$. But we can divide each coefficient of $w$ by $X_{1} X_{2} X_{3}$ to obtain a non-zero cycle $z \in Z_{j}\left(\mathfrak{m}^{c}\right)$ with coefficients of degree $c-3$. It follows that $S^{(c)}$ does not satisfy the property $N_{3 c-2}$. This concludes the proof for $n=3$.

Now let $n>3$. Recall that $H_{i}\left(\mathfrak{m}^{c}\right)$ is multigraded. For a vector $a=\left(a_{1}, \ldots, a_{n}\right) \in \mathbb{N}^{n}$ with $a_{i}=0$ for $i>3$ let $b=\left(a_{1}, a_{2}, a_{3}\right)$. We may identify

$$
H_{i}\left(\mathfrak{m}^{c}\right)_{a}=H_{i}\left(\mathfrak{m}_{3}^{c}\right)_{b}
$$

where $H_{i}\left(\mathfrak{m}_{3}^{c}\right)$ is the corresponding Koszul homology in 3 variables. Since for $n=3$ the $c$-th Veronese does not satisfy $N_{3 c-2}$ it follows that the same is true for all $n \geq 3$, proving that index $\left(S^{(c)}\right) \leq 3 c-3$.

Remark 4.8. It is well-known that $\operatorname{reg} S^{(c)} \leq n-1$ in general, i.e., $t_{i}\left(S^{(c)}\right) \leq i+n-$ 1. Analogously to the proof of Theorem 4.7 one can determine the largest $i$ such that $t_{i}\left(S^{(c)}\right)<i+n-1$. Again this is determined by elements in $Z_{i}\left(\mathfrak{m}^{c}\right)$ with coefficients of degree $c-n$. It remains to count the monomials of $S$ in that degree. For example, for $c \geq n=4$ one obtains $t_{i}\left(S^{(c)}\right)<i+3$ if and only if $i \leq 2 c^{2}-2$.

\section{THE GREEN-LAZARSFELD INDEX OF VERONESE SUBRINGS OF STANDARD GRADED RINGS}

Let $D$ be a Koszul $K$-algebra and $I$ be a homogeneous ideal of $D$. Set $R=D / I$. We want to relate the Green-Lazarsfeld index of $R^{(c)}$ to that of $D^{(c)}$. For a polynomial ring $S$ Aramova, Bărcănescu and Herzog proved in [2, Theorem 2.1] that the Veronese modules $V_{S}(c, k)$ have a linear resolution over the Veronese ring $S^{(c)}$. We show that this property holds for Koszul algebras in general.

Lemma 5.1. Assume $D$ is a Koszul algebra and, for a given $c$, let $T=\operatorname{Sym}\left(D_{c}\right)$ be the symmetric algebra of $D_{c}$.

(a) The Veronese module $V_{D}(c, k)$ has a linear resolution as a $D^{(c)}$-module.

(b) For every $k=0, \ldots, c-1$ we have

$$
t_{i}^{T}\left(V_{D}(c, k)\right) \leq t_{i}^{T}\left(D^{(c)}\right) .
$$

Proof. (a) Let $\mathfrak{m}$ denote the homogeneous maximal ideal of $D$, and set $A=D^{(c)}$ and $V_{k}=V_{D}(c, k)$. We prove by induction on $i$ that $t_{i}^{A}\left(V_{k}\right) \leq i$ for all $i$ and $k$. For $i=0$ the assertion is obvious and it is so for $k=0$ and $i \geq 0$, too. Assume that $i>0$. The ideal $\mathfrak{m}^{k}$ is generated in degree $k$ and, since $D$ is Koszul, it has a linear resolution over $D$. Shifting that resolution by $k$, we obtain a complex

$$
\cdots \rightarrow F_{i} \rightarrow F_{i-1} \rightarrow \cdots \rightarrow F_{1} \rightarrow F_{0} \rightarrow 0
$$

resolving $\mathfrak{m}^{k}(k)$ and such that $F_{i}=D(-i)^{\beta_{i}}$. Applying the exact functor ()$^{(c)}$ to it we get an exact complex of $A$-modules

$$
\cdots \rightarrow F_{i}^{(c)} \rightarrow F_{i-1}^{(c)} \rightarrow \cdots \rightarrow F_{1}^{(c)} \rightarrow A^{\beta_{0}} \rightarrow V_{k} \rightarrow 0 .
$$

Note that $D(-j)^{(c)}=V_{e}(-\lceil j / c\rceil)$ where $e=c\lceil j / c\rceil-j$. Therefore $F_{j}^{(c)}=V_{e_{j}}(-\lceil j / c\rceil)^{\beta_{j}}$ where $e_{j}=c\lceil j / c\rceil-j$. Applying Lemma2.2(b) to the complex above we have

$$
t_{i}^{A}\left(V_{k}\right) \leq \max \left\{t_{i-j}^{A}\left(V_{e_{j}}\right)+\lceil j / c\rceil: j=0, \ldots, i\right\} .
$$


Obviously $t_{i}^{A}\left(V_{e_{0}}\right)=t_{i}^{A}(A)=-\infty$ and, by induction, $t_{i-j}^{A}\left(V_{e_{j}}\right) \leq i-j$ for $j=1, \ldots, i$. Therefore

$$
t_{i}^{A}\left(V_{k}\right) \leq \max \{i-j+\lceil j / c\rceil: j=1, \ldots, i\}=i
$$

and this concludes the proof of (a). For (b) we may apply Lemma 2.2 (b) to the minimal $A$-free resolution of $V_{k}$ and to get the desired inequality.

Now we prove the main result of this section.

Theorem 5.2. Assume $D$ is a Koszul algebra and $R=D /$ I. Let $c \geq \operatorname{slope}_{D}(R)$. Then index $\left(R^{(c)}\right) \geq \operatorname{index}\left(D^{(c)}\right)$.

Proof. To prove the statement we set $A=D^{(c)}$ and $B=R^{(c)}$. By virtue of Lemma 2.2 (d) it is enough to show that $\operatorname{reg}_{A}(B)=0$. Let

$$
\cdots \rightarrow F_{p} \rightarrow \cdots \rightarrow F_{1} \rightarrow F_{0} \rightarrow R \rightarrow 0
$$

be the minimal graded free resolution of $R$ over $D$. Since ()$^{(c)}$ is an exact functor, we obtain an exact complex of finitely generated graded $A$-modules

$$
\cdots \rightarrow F_{p}^{(c)} \rightarrow \cdots \rightarrow F_{1}^{(c)} \rightarrow F_{0}^{(c)} \rightarrow B \rightarrow 0 .
$$

Hence by virtue of Lemma2.2 (b) we have

$$
\operatorname{reg}_{A}(B) \leq \max \left\{\operatorname{reg}_{A}\left(F_{i}^{(c)}\right)-i: i \geq 0\right\} .
$$

Note that $D(-k)^{(c)}=V_{D}(c, e)(-\lceil k / c\rceil)$ where $e=c\lceil k / c\rceil-k$. Hence, by virtue of Lemma 5.1, $\operatorname{reg}_{A}\left(D(-k)^{(c)}\right)=\lceil k / c\rceil$. Therefore, since $F_{i}=\bigoplus_{k \in \mathbb{Z}} S(-k)^{\beta_{i k}^{D}(R)}$ we get $\operatorname{reg}_{A}\left(F_{i}^{(c)}\right)=$ $\left\lceil t_{i}^{D}(R) / c\right\rceil$. Summing up,

$$
\operatorname{reg}_{A}(B) \leq \max \left\{\left\lceil t_{i}^{D}(R) / c\right\rceil-i: i \geq 0\right\} .
$$

If $c \geq$ slope $_{D}(R)$, then $t_{i}^{D}(R) \leq c i$ and hence $\operatorname{reg}_{A}(B)=0$. This concludes the proof.

As a corollary we have:

Corollary 5.3. Let $S$ be a polynomial ring and $R=S / I$ a standard graded algebra quotient of it and let $c \geq \operatorname{slope}_{S}(R)$. Then $\operatorname{index}\left(R^{(c)}\right) \geq \operatorname{index}\left(S^{(c)}\right)$. In particular,

(a) index $\left(R^{(c)}\right) \geq c$. Furthermore, if $K$ has characteristic 0 or $>c+1$, then we have index $\left(R^{(c)}\right) \geq c+1$.

(b) If $\operatorname{dim} R_{1}=3$, then index $\left(R^{(c)}\right) \geq 3 c-3$.

Note that $\operatorname{slope}_{S}(R)=2$ if $R$ is Koszul; see [4]. Furthermore $\operatorname{slope}_{S}(R) \leq a$ if $R$ is defined by either a complete intersection of elements of degree $\leq a$ or by a Gröbner basis of elements of degree $\leq a$.

Remark 5.4. Sometimes the bound in Theorem 5.2 can be improved by a more careful argumentation. Let $R=S / I$ and let $T$ be the symmetric algebra of $S_{c}$. For instance, using the argument of the proof of Theorem 5.2 one shows that

$$
\left.t_{i}^{T}\left(R^{(c)}\right) \leq \max \left\{t_{i-j}^{T}\left(S^{(c)}\right)+\left\lceil t_{j}^{S}(R) / c\right\rceil\right\}: j=0,1, \ldots, i\right\} .
$$


It follows that index $\left(R^{(c)}\right) \geq p$ if $c \geq p$ and index $(R) \geq p$, a result proved by Rubei in [26]. It is very easy to show that $R^{(c)}$ is defined by quadrics, i.e. $\operatorname{index}\left(R^{(c)}\right) \geq 1$ provided $c \geq t_{1}^{S}(R) / 2$. Similarly, one can prove that index $\left(R^{(c)}\right) \geq p$ if

$$
c \geq \max \left\{p, \max \left\{t_{j}^{S}(R) / j: j=1, \ldots, p-1\right\}, t_{p}^{S}(R) /(p+1)\right\} .
$$

\section{Remark 5.5.}

(a) Let us say that a positively graded $K$-algebra is almost standard if $R$ is Noetherian and a finitely generated module over $K\left[R_{1}\right]$. If $K$ is infinite, then this property is equivalent to the existence of a Noether normalization generated by elements of degree 1. Galliego and Purnaprajna [15, Theorem 1.3] proved a general result on the property $N_{p}$ of Veronese subalgebras of almost standard $K$-algebras $R$ of depth $\geq 2$ over fields of characteristic $0: R^{(c)}$ has $N_{p}$ for all $c \geq \max \{\operatorname{reg}(R)+p-$ $1, \operatorname{reg}(R), p\}$. If $\operatorname{reg}(R) \geq 1$ and $p \geq 1$, this amounts to the property $N_{c-\operatorname{reg}(R)+1}$ of $R^{(c)}$ for all $c \geq \operatorname{reg}(R)$. Thus Theorem 5.2 gives a stronger result for standard graded algebras.

(b) Eisenbud, Reeves and Totaro [12] proved that the Veronese subalgebras $R^{(c)}$ of standard graded $K$-algebra $R$ are defined by an ideals with Gröbner bases of degree 2 for all $c \geq(\operatorname{reg}(R)+1) / 2$. It follows that these algebras are Koszul.

(c) If $R$ is almost standard and Cohen-Macaulay, then $R^{(c)}$ is defined by an ideal with a Gröbner bases of degree 2 for every $c \geq \operatorname{reg}(R)$. See Bruns, Gubeladze and Trung [7. Theorem 1.4.1] or Bruns and Gubeladze [6, Theorem 7.41].

\section{THE MULTIGRADED CASE}

The results presented in this paper have natural extensions to the multigraded case. Here we just formulate the main statements. Detailed proofs will be given in the forthcoming article [9]. Suppose $S=K\left[X^{(1)}, \ldots, X^{(m)}\right]$ is a $\mathbb{Z}^{m}$-graded polynomial ring in which each $X^{(i)}$ is the set of variables of degree $e_{i} \in \mathbb{Z}^{m}$. For a vector $c \in\left(c_{1}, \ldots, c_{m}\right) \in \mathbb{N}_{+}^{m}$ consider the $c$-th diagonal subring $S^{(c)}=\oplus_{i \in \mathbb{N}} S_{i c}$, the coordinate ring of the corresponding Segre-Veronese embedding. The following result improves the bound of Hering, Schenck and Smith [21] by one:

Theorem 6.1. With the notation above one has: $\min (c) \leq \operatorname{index}\left(S^{(c)}\right)$. Moreover, we have $\min (c)+1 \leq \operatorname{index}\left(S^{(c)}\right)$ if $\operatorname{char} K=0$ or $\operatorname{char} K>1+\min (c)$.

Similarly one has the multigraded analog of Theorem 5.2. Here one uses the fact, proved in [11], given any $\mathbb{Z}^{m}$-graded standard graded algebra quotient of $S$ then if the $c_{j}$ 's are big enough (in terms of the multigraded Betti numbers of $R$ over $S$ ) then $\operatorname{reg}_{S^{(c)}}\left(R^{(c)}\right)=$ 0 .

Proposition 6.2. Assume that for all $j=1 \ldots, m$ one has $c_{j} \geq \max \left\{\alpha_{j} / i: i>0, \alpha \in\right.$ $\mathbb{Z}^{m}$ and $\left.\beta_{i, \alpha}^{S}(R) \neq 0,\right\}$ then $\operatorname{index}\left(R^{(c)}\right) \geq \operatorname{index}\left(S^{(c)}\right)$.

\section{REFERENCES}

[1] J. L. Andersen, Determinantal rings associated with symmetric matrices: a counterexample. Ph.D. Thesis, University of Minnesota (1992). 
[2] A. Aramova, S. Bărcănescu and J. Herzog, On the rate of relative Veronese submodules. Rev. Roum. Math. Pures Appl. 40, 243-251 (1995).

[3] L. L. Avramov, Infinite free resolutions. In: Elias, J. et al. (ed.), Six lectures on commutative algebra, Birkhäuser, Prog. Math. 166, 1-118 (1998).

[4] L. L. Avramov, A. Conca and S. Iyengar, Resolutions of Koszul algebra quotients of polynomial rings. arXiv:0904.2843, to appear in Math. Res. Lett.

[5] L. L. Avramov and D. Eisenbud, Regularity of modules over a Koszul algebra. J. Algebra 153, 85-90 (1992).

[6] W. Bruns and J. Gubeladze, Polytopes, rings, and K-theory. Springer 2009.

[7] W. Bruns, J. Gubeladze and N. V. Trung, Normal polytopes, triangulations, and Koszul algebras. J. Reine Angew. Math. 485, 123-160 (1997).

[8] W. Bruns and J. Herzog, Cohen-Macaulay rings. Rev. ed. Cambridge University Press 1998.

[9] W. Bruns, A. Conca and T. Römer, Koszul cycles, in preparation (2009).

[10] CoCoATeam, CoCoA: a system for doing Computations in Commutative Algebra. Available at http://cocoa.dima.unige.it.

[11] A. Conca, J. Herzog, N. V. Trung and G. Valla, Diagonal subalgebras of bigraded algebras and embeddings of blow-ups of projective spaces. Amer. J. Math. 119, 859-901 (1997).

[12] D. Eisenbud, A. Reeves and B. Totaro, Initial ideals, Veronese subrings, and rates of algebras. Adv. in Math. 109, 168-187 (1994).

[13] D. Eisenbud, M. Green, K. Hulek and S. Popescu, Restricting linear syzygies: algebra and geometry. Compos. Math. 141, 1460-1478 (2005).

[14] D. Eisenbud, The geometry of syzygies, a second course in commutative algebra and algebraic geometry. Graduate Texts in Mathematics 229, Springer 2005.

[15] F. J. Gallego and B. P. Purnaprajna, Projective normality and syzygies of algebraic surfaces. J. Reine Angew. Math. 506, 145-180 (1999); erratum ibid. 523, 233-234 (2000).

[16] D. R. Grayson and M.E. Stillman, Macaulay 2, a software system for research in algebraic geometry. Available at http://www .math.uiuc .edu/Macaulay2/.

[17] M. L. Green, Koszul cohomology and the geometry of projective varieties. II. J. Differ. Geom. 20 , 279-289 (1984).

[18] M. L. Green and R. Lazarsfeld, On the projective normality of complete linear series on an algebraic curve. Invent. Math. 83, 73-90 (1986).

[19] M. L. Green and R. Lazarsfeld, Some results on the syzygies of finite sets and algebraic curves. Compos. Math. 67, 301-314 (1988).

[20] G. M. Greuel, G. Pfister and H. Schönemann, Singular 3.0 A Computer Algebra System for Polynomial Computations. Centre for Computer Algebra, University of Kaiserslautern (2001). Available at http://www.singular. uni-kl.de.

[21] M. Hering, H. Schenck and G.G.Smith, Syzygies, multigraded regularity and toric varieties. Compos. Math. 142, 1499-1506 (2006).

[22] T. Jozefiak, P. Pragacz and J. Weyman, Resolutions of determinantal varieties and tensor complexes associated with symmetric and antisymmetric matrices. Astérisque 87-88, 109-189 (1981).

[23] G. Ottaviani and R. Paoletti, Syzygies of Veronese embeddings. Compos. Math. 125, 31-37 (2001).

[24] E. Park, Some effects of Veronese map on syzygies of projective varieties. arXiv:math/0509710

[25] T. Römer, Bounds for Betti numbers. J. Algebra 249, 20-37 (2002).

[26] E. Rubei, A note on property $\mathrm{N}_{p}$. Manuscripta Math. 101, 449-455 (2000).

[27] E. Rubei, A strange example on Property $\mathrm{N}_{p}$. Manuscripta Math. 108, 135-137 (2002).

[28] E. Rubei, A result on resolutions of Veronese embeddings. Ann. Univ. Ferrara, Nuova Ser., Sez. VII 50, 151-165 (2004).

[29] J. Weyman, Cohomology of vector bundles and syzygies. Cambridge University Press 2003. 
UNIVERSitÄt OSNABRÜCK, INSTITUT FÜR MATHEMATIK, 49069 OSNABRÜCK, GERMANY

E-mail address: wbruns@uos.de

Dipartimento di Matematica, Universitá di Genova, Via Dodecaneso 35, 16146 GenOVA, ITALY

E-mail address: conca@dima. unige.it

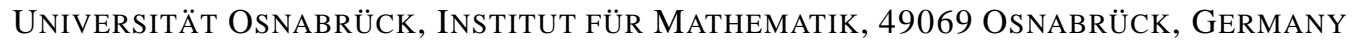

E-mail address: troemer@uos.de 